\begin{tabular}{cc}
\hline & COMPUTATIONAL RESEARCH PROGRESS IN APPLIED SCIENCE \& ENGINEERING (CRPASE) \\
\hline
\end{tabular}

\title{
On Maximum Likelihood Estimators of the Parameters of Three-Parameters Weibull Distribution Using Different Ranked Set Sampling Schemes
}

\author{
Essam Fawzy Aziz ${ }^{1}$, Mostafa Shaaban ${ }^{2 *}$ \\ ${ }^{1}$ Department of Statistics, Faculty of Commerce, Ain Shams University, Cairo, Egypt \\ ${ }^{2}$ The High Institute for Tourism, Hotels and Computer, El-Seyouf, Alexandria, Egyp
}

\begin{tabular}{|c|c|}
\hline Keywords & Abstract \\
\hline $\begin{array}{l}\text { Simple Random Sampling, } \\
\text { Ranked Set Sampling, } \\
\text { Extreme Ranked Set } \\
\text { Sampling, } \\
\text { Double Ranked Set } \\
\text { Sampling, } \\
\text { Estimation Parameter. }\end{array}$ & $\begin{array}{l}\text { Al-Saleh and Al-Kadiri first proposed double rank set sampling (DRSS). It seems that this } \\
\text { ranked set sampling (RSS) modification can reduce the loss of RSS efficiency caused by } \\
\text { ranking errors, and it is more effective than RSS and simple random sampling (SRS) to } \\
\text { estimate the population mean. The proposed likelihood function is used to estimate the } \\
\text { parameters of the three-Parameters Weibull distribution. Based on double ranked set } \\
\text { sampling, extreme ranked set sampling, ranked set sampling (RSS) and simple random } \\
\text { sampling (SRS) designs, the maximum likelihood estimator (MLE) is compared with the } \\
\text { corresponding likelihood estimator. A simulation was carried out and the absolute relative } \\
\text { biases, mean square error (MSE) and relative efficiency of different schemes were } \\
\text { compared. It is found that, MSEs based SRS data has the largest MSEs comparing to RSS } \\
\text { and its modifications schemes. This study revealed that DRSS technique has the superior } \\
\text { over the rest of other sampling schemes. In almost all cases, DRSS has the smallest MSEs } \\
\text { and largest efficiencies. }\end{array}$ \\
\hline
\end{tabular}

\section{Introduction}

The Weibull distribution is widely used in reliability and lifetime studies and proved an appropriate fit for most life data, except for data with non-monotonic empirical hazards. This type of data is often encountered in survival analysis, which makes it impossible for the Weibull model to analyze it. In many applications, $\alpha$ is assumed known (often $\alpha=0$ ), for which results [1] guarantee the existence of a unique maximum likelihood estimator $\lambda, \beta$, and [2] the first of these is introduced tree parameter Weibull and concerned with asymptotic theory for maximum likelihood estimators. The cumulative distribution

function (CDF) of the three-parameter Weibull distribution is given by

$$
F(x ; \lambda, \beta, \alpha)=1-e^{-\lambda(x-\alpha)^{\beta}}
$$

where $\lambda>0, \beta>0$ and $\alpha<x$. The parameters $\lambda, \beta$ and $\alpha$ are known as the scale, shape and location parameters, respectively. The corresponding probability density function $(\mathrm{PDF})$ is

$$
f(x ; \lambda, \beta, \alpha)=\lambda \beta(\mathrm{x}-\alpha)^{\beta-1} e^{-\lambda(\mathrm{x}-\alpha)^{\beta}}
$$

and the two parameters Weibull distribution is a special case of (2) when $\alpha=1$.

\section{Some Ranked Set Sampling Techniques}

In this section, various sampling procedures for selection of units in the sample will be considered; brief descriptions of ranked set sampling (RSS), extreme ranked set sampling (ERSS) and double extreme ranked set sampling (DRSS) schemes will be introduced.

McIntyre, 1952 [3] proposed Ranked Set Sampling (RSS) to improve the estimation of the population mean, and

\footnotetext{
* Corresponding Author: Mostafa Shaaban

E-mail address: moushaaban@gmail.com
} 
the RSS method constitutes the sampling unit, because some measurements are not performed, the possibility of ranking errors increases. In order to overcome this problem, various modifications to RSS have been proposed, for example:[4] introduced extreme ranking set sampling (ERSS), [5] proposed median ranking set sampling (MRSS), The twostage RSS design developed Double Ranked Set Sampling (DRSS) [6]. In addition to these studies, some authors have considered using RSS or its modification to estimate the parameters of a known distribution.

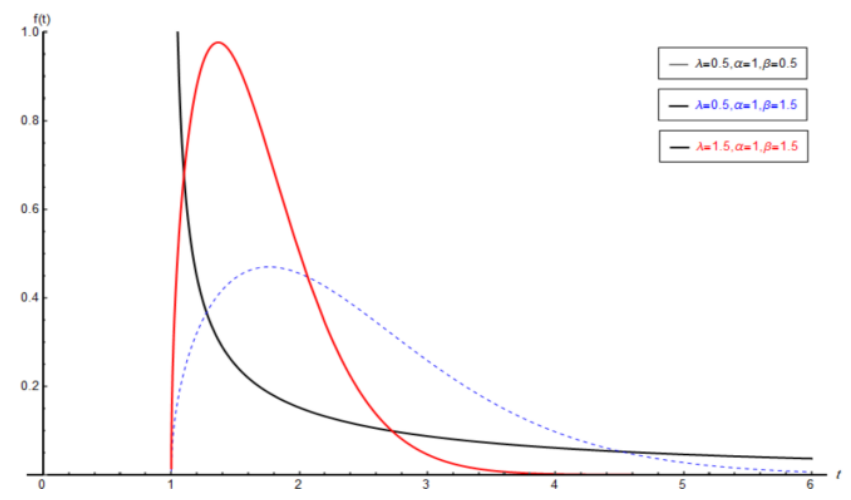

Figure 1. PDF of the EPGW distribution for different parameter values

For example, [7] studied Weibull and Pareto distributions, [8] introduced the maximum likelihood estimation of the modified Weibull distribution parameters using extreme ranking set sampling, [9], [10] and [11].and a $k$-stage RSS design uses $n^{k+1}$ sample units from the target population to produce a sample of size $n$ after $k$ stages of ranking developed [12]. For more details, see [13], [14], [15] and [16], see more [17], [18] and [19].

\subsection{Ranked Set Sampling}

McIntyre proposed RSS technique as a useful procedure, the cost of quantifying all sampling units was high, but according to the characteristics of the survey, a small set of units can be easily ranked. Without actual quantification. The ranking criterion may be based on the value of accompanying variables or personal judgment, for example. A number of studies have proved that RSS is more efficient in estimating a large number of population parameters than SRS.

The RSS scheme can be described as follows:

Step 1: Randomly select $m^{2}$ sample units from the population.

Step 2: Allocate the $\mathrm{m} 2$ selected units as randomly as possible into $\mathrm{m}$ sets, each of size $\mathrm{m}$.

Step 3: By including the smallest ordered unit in the first group and the second smallest ordered unit in the second group, the sample is determined for actual quantification, This process will continue until the largest is selected from the last group The unit of sorting.

Step 4: Repeat steps 1 through 4 for $r$ cycles to obtain a sample of size $m r$.

\begin{tabular}{|c|c|c|c|c|c|c|c|c|}
\hline \multicolumn{3}{|c|}{ cycle 1} & \multicolumn{3}{|c|}{ cycle 2} & \multicolumn{3}{|c|}{ cycle 3} \\
\hline$X_{(11) 1}$ & $X_{(12) 1}$ & $X_{(13) 1}$ & $X_{(11) 2}$ & $X_{(12) 2}$ & $X_{(13) 2}$ & $X_{(11) 3}$ & $X_{(12) 3}$ & $X_{(13) 3}$ \\
\hline$X_{(21) 1}$ & $X_{(22) 1}$ & $X_{(23) 1}$ & $X_{(21) 2}$ & $X_{(22) 2}$ & $X_{(23) 2}$ & $X_{(21) 3}$ & $X_{(22) 3}$ & $X_{(23) 3}$ \\
\hline$X_{(31) 1}$ & $X_{(32) 1}$ & $X_{(33) 1}$ & $X_{(31) 2}$ & $X_{(32) 2}$ & $X_{(33) 2}$ & $X_{(31) 3}$ & $X_{(32) 3}$ & $X_{(33) 3}$ \\
\hline
\end{tabular}

Figure 2. RSS design

Let $\left\{X_{(i i) s}, i=1,2, \ldots, m ; s=1,2, \ldots, r\right\}$ be a ranked set sample where $m$ is the set size and $r$ is the number of cycles. Then the probability density function (PDF) of $X_{(i i) s}$ is given by

$$
f_{i}\left(x_{(i i) j}\right)=C_{1}\left[F\left(x_{(i i) j}\right)\right]^{i-1} \cdot\left[1-F\left(x_{(i i) j}\right)\right]^{m-i} f\left(x_{(i i) j}\right)
$$

where $C_{1}=\frac{m !}{(i-1) !(m-1) !},,-\infty<x_{(i i) j}<\infty$,

using Eq. (3) the likelihood function corresponding to RSS scheme is given by:

$$
\begin{gathered}
L_{R S S}(\theta \mid x)=\prod_{j=1}^{r} \prod_{i=1}^{m} C_{1} f\left(x_{(i i) j} ; \theta\right)\left[F\left(x_{(i i) j} ; \theta\right)\right]^{i-1} \\
\cdot\left[1-F\left(x_{(i i) j} ; \theta\right)\right]^{m-i}
\end{gathered}
$$

\subsection{Extreme Ranked Set Sampling}

[4] introduced a modification of McIntyre's RSS scheme to produce other sampling schemes the extreme ranked set sampling (ERSS) which does not need a complete ranking as for RSS. They investigated ERSS by quantifying the smallest and largest order statistics instead of detailed ranking. The ERSS procedure can be summarized as follows

Step (1): Select $m$ random samples of size $m$ units from the population.

Step (2): Rank the units within each sample with respect to a variable of interest by visual inspection or any other cost free method.

Step (3): If the sample size $n$ is odd, select from $\frac{m-1}{2}$ samples the smallest unit, from the other $\frac{m-1}{2}$ the largest unit and for the last sample select the median of the sample for actual measurement.

$$
\begin{aligned}
& {\left[\begin{array}{cccccc}
x_{(11) j} & x_{(12) j} & \cdots & x_{\left(1 \frac{m+1}{2}\right) j} & \cdots & x_{(1 m) j} \\
\vdots & \vdots & \vdots & \vdots & \vdots & \vdots \\
x_{\left(\frac{m-1}{2} 1\right) j} & x_{\left(\frac{m-1}{2} 2\right) j} & \cdots & \left.x_{\left(\frac{m-1}{2}\right.} \frac{m+1}{2}\right) j & \cdots & x_{\left(\frac{m-1}{2} m\right) j} \\
x_{\left(\frac{m+1}{2} 1\right) j} & x_{\left(\frac{m+1}{2} 2\right) j} & \cdots & x_{\left(\frac{m+1}{2} \frac{m+1}{2}\right) j} & \cdots & x_{\left(\frac{m+1}{2} m\right) j} \\
\vdots & \vdots & \vdots & \vdots & \vdots & \vdots \\
x_{(m-11) j} & x_{(m-12) j} & \cdots & \left.x_{(m-1} \frac{m+1}{2}\right) j & \cdots & x_{(m-1 m) j} \\
x_{(m 1) j} & x_{(m 2) j} & \cdots & x_{\left(m \frac{m+1}{2}\right) j} & \cdots & x_{(m m) j}
\end{array}\right]} \\
& \stackrel{\text { for modd and r times }}{\longrightarrow}\left[\begin{array}{cccccc}
x_{(11) j} & x_{(12) j} & \cdots & x_{\left(1 \frac{m+1}{2}\right) j} & \cdots & x_{(1 m) j} \\
\vdots & \vdots & \vdots & \vdots & \vdots & \vdots \\
x_{\left(\frac{m-1}{2} 1\right) j} & x_{\left(\frac{m-1}{2} 2\right) j} & \cdots & x_{\left(\frac{m-1}{2} \frac{m+1}{2}\right) j} & \cdots & x_{\left(\frac{m-1}{2} m\right) j} \\
x_{\left(\frac{m+1}{2}\right) j} & x_{\left(\frac{m+1}{2} 2\right) j} & \cdots & \left.x_{\left(\frac{m+1}{2}\right.} \frac{m+1}{2}\right) j & \cdots & x_{\left(\frac{m+1}{2} m\right) j} \\
\vdots & \vdots & \vdots & \vdots & \vdots & \vdots \\
x_{(m-11) j} & x_{(m-12) j} & \cdots & \left.x_{(m-1} \frac{m+1}{2}\right) j & \cdots & x_{(m-1 m) j} \\
x_{(m 1) j} & x_{(m 2) j} & \cdots & x_{\left(m \frac{m+1}{2}\right) j} & \cdots & x_{(m m) j}
\end{array}\right]
\end{aligned}
$$

Figure 3. ERSS Design in Case of Odd Sample Size

The PDFs of the ERSS in case of odd sample size will be as follows: 


$$
\begin{aligned}
f_{m}\left(x_{(i 1) j}\right)=m f\left(x_{(i 1) j} ; \theta\right)\left[1-F\left(x_{(i 1) j} ; \theta\right)\right]^{m-1}, & \\
-\infty<x_{(i 1) j} & <\infty
\end{aligned}
$$

where $1 \leq i \leq q, q=\frac{m-1}{2}$

$$
\begin{array}{r}
f_{m}\left(x_{(i m) j}\right)=m\left[f\left(x_{(i m) j} ; \theta\right)\right]^{n-1}\left[1-F\left(x_{(i m) j} ; \theta\right)\right]^{m-1}, \\
-\infty<x_{(i m) j}<\infty .
\end{array}
$$

where $q+1 \leq i \leq m-1$, and

$$
\begin{aligned}
f_{m}\left(x_{\left(m \frac{m+1}{2}\right) j}\right) & =\frac{m !}{\left[\left(\frac{m+1}{2}-1\right) !\right]^{2}} f\left(x_{\left(m \frac{m+1}{2}\right) j} ; \theta\right) \\
\cdot\left(F\left(x_{\left(m \frac{m+1}{2}\right) j} ; \theta\right)\right. & \left.\left(1-F\left(x_{\left(m \frac{m+1}{2}\right) j} ; \theta\right)\right)\right)^{\frac{m-1}{2}} \\
& -\infty<x_{\left(m \frac{m+1}{2}\right) j}<\infty .
\end{aligned}
$$

where $x_{(i 1) j}$ is the smallest order statistic from the $i^{\text {th }}$ set of the $j^{\text {th }}$ cycle, $x_{(\mathrm{im}) j}$ is the largest order statistic from the $i^{t h}$ set of the $j^{\text {th }}$ cycle and $x_{\left(m \frac{m+1}{2}\right) j}$ is the median order statistic from the $m^{\text {th }}$ set of the $j^{\text {th }}$ cycle.

The Likelihood function corresponding to ERSS scheme for $m$ is odd and with $r$ cycles is given as follows:

$$
\begin{aligned}
& L_{E R S S}(\theta) \propto\left[\prod_{j=1}^{r} \prod_{i=1}^{q} m f\left(x_{(i 1) j} ; \theta\right)\left[F\left(x_{(i 1) j} ; \theta\right)\right]^{m-1}\right] \\
& \cdot\left[\prod_{j=1}^{r}\left[\prod_{i=q+1}^{m-1} m f\left(x_{(i m) i, j} ; \theta\right)\left[1-F\left(x_{(i m) j} ; \theta\right)\right]^{m-1}\right] .\right. \\
& {\left[f\left(x_{\left(m \frac{m+1}{2}\right) j} ; \theta\right)\left(F\left(x_{\left(m \frac{m+1}{2}\right) j} ; \theta\right)\left(1-F\left(x_{\left(\frac{m+1}{2} m\right) j} ; \theta\right)\right)\right)^{\frac{m-1}{2}}\right] }
\end{aligned}
$$

Step (4): If the sample size is even, select from $\frac{m}{2}$ samples the smallest unit and from the other $\frac{m}{2}$ samples the largest unit for actual measurement.

$\left[\begin{array}{cccc}x_{(11)}^{(1)} & x_{(12)}^{(1)} & \ldots & x_{(1 \mathrm{~m})}^{(1)} \\ \vdots & \vdots & \vdots & \vdots \\ x_{\left(\frac{m}{2} 1\right)}^{(1)} & x_{\left(\frac{m}{2} 2\right)}^{(1)} & \cdots & x_{\left(\frac{m}{2} m\right)}^{(1)} \\ x_{\left(\frac{m}{2}+11\right)}^{(1)} & x_{\left(\frac{m}{2}+12\right)}^{(1)} & \cdots & x_{\left(\frac{m}{2}+1 \mathrm{~m}\right)}^{(1)} \\ \vdots & \vdots & \vdots & \vdots \\ x_{(\mathrm{m} 1)}^{(1)} & x_{(\mathrm{m} 2)}^{(1)} & \cdots & x_{(\mathrm{m} \mathrm{m})}^{(1)}\end{array}\right]$

$\stackrel{\text { for m even and r times }}{\longrightarrow}\left[\begin{array}{ccccc}x_{(1)}^{(1)} & x_{(12)}^{(1)} & \ldots & x_{(1 \mathrm{~m})}^{(1)} \\ \vdots & \vdots & \vdots & \vdots \\ x_{\left(\frac{m}{2} 1\right)}^{(1)} & x_{\left(\frac{m}{2} 2\right)}^{(1)} & \cdots & x_{\left(\frac{m}{2} m\right)}^{(1)} \\ x_{\left(\frac{m}{2}+11\right)}^{(1)} & x_{\left(\frac{m}{2}+12\right)}^{(1)} & \cdots & x_{\left(\frac{m}{2}+1 \mathrm{~m}\right)}^{(1)} \\ \vdots & \vdots & \vdots & \vdots \\ x_{(\mathrm{m} 1)}^{(1)} & x_{(\mathrm{m} 2)}^{(1)} & \cdots & x_{(\mathrm{m} \mathrm{m})}^{(1)}\end{array}\right]$

Figure 4. ERSS Design in Case of Even Sample Size.

The PDFs of the ERSS in case of even sample size are defined as in Eq. (5) and Eq. (6) and the Likelihood function corresponding to ERSS scheme for even set sizes $(m=2 p)$ and with $r$ cycles introduced by [8] is given as follows:

$$
\begin{aligned}
& L_{E R S S}(\theta) \propto\left[\prod_{j=1}^{r} \prod_{i=1}^{p} m f\left(x_{(i 1) j} ; \theta\right)\left[1-F\left(x_{(1 i) j} ; \theta\right)\right]^{m-1}\right] . \\
& {\left[\prod_{j=1}^{r} \prod_{i=p+1}^{m} m f\left(x_{(i m) j} ; \theta\right)\left[F\left(x_{(i m) i, j} ; \theta\right)\right]^{m-1}\right]}
\end{aligned}
$$

Step (5): The cycle may be repeated $r$ times to get $n=m r$ units from ERSS data.

\subsection{Double Ranked Set Sampling}

The best known multistage RSS procedure is DRSS design. DRSS is a two stage design was proposed by [6], which can be detailed as follows:

Step 1: Select $m^{3}$ elements from the target population and divide these elements randomly into $m$ sets (of size $m^{2}$ ).

Step 2: Select a sample of size $m$ in each set using RSS method.

Step 3: Apply the RSS procedure again to elements selected in step 2 to obtain a DRSS of size $m$.

Step 4: The cycle may be repeated $r$ times.

$$
\begin{gathered}
{\left[\begin{array}{ccc}
x_{(11) 1} & \cdots & x_{(1 m) 1} \\
\vdots & \ddots & \vdots \\
x_{(m 1) 1} & \cdots & x_{(m m) 1}
\end{array}\right],\left[\begin{array}{ccc}
x_{(11) 2} & \cdots & x_{(1 m) 2} \\
\vdots & \ddots & \vdots \\
x_{(m 1) 2} & \cdots & x_{(m m) 2}
\end{array}\right],\left[\begin{array}{ccc}
x_{(11) 3} & \cdots & x_{(1 m) 3} \\
\vdots & \ddots & \vdots \\
x_{(m 1) 3} & \cdots & x_{(m m) 3}
\end{array}\right]} \\
\text { and } \cdots\left[\begin{array}{ccc}
x_{(11) m} & \cdots & x_{(1 m) m} \\
\vdots & \ddots & \vdots \\
x_{(m 1) m} & \cdots & x_{(m m) m}
\end{array}\right]
\end{gathered}
$$

Figure 5. DRSS design in case of odd sample size

So, we have four judgment ranked sets of size $m$ each:

$$
\begin{aligned}
& X_{1, j}=\min \left\{\left\{x_{(11)}^{(\mathrm{j})}, x_{(22)}^{(\mathrm{j})}, \ldots . x_{(\mathrm{mm})}^{(\mathrm{j})}\right\}, j=1,2, \ldots, r\right\}, \\
& X_{m, k}=\max \left\{\left\{x_{(11)}^{(\mathrm{k})}, x_{(22)}^{(\mathrm{k})}, \ldots . x_{(\mathrm{mm})}^{(\mathrm{k})}\right\}, k=r+2, \ldots, m\right\}, \\
& \text { and } \\
& X_{(r+1),(r+1)}=\operatorname{median}\left\{\left\{X_{(1, r+1)}, X_{(2, r+1)}, \ldots . X_{(m, r+1)}\right\}\right\} .
\end{aligned}
$$

The likelihood function corresponding to DRSS scheme that proposed by [9] is given as follows:

\section{Case I: $m$ even $(m=2 r)$}




$$
\begin{aligned}
& L(\theta) \\
& =\left[\prod_{j=1}^{r} m f_{1: m}\left(x_{1, j}\right)\left[1-F_{1: m}\left(x_{1, j}\right)\right]^{m-1}\right] \\
& \cdot\left[\prod_{k=r+1}^{m} m f_{m: m}\left(x_{m, k}\right)\left[F_{m: m}\left(x_{m, k}\right)\right]^{m-1}\right]
\end{aligned}
$$

where

$$
\begin{aligned}
& f_{1: m}\left(x_{j}\right)=m f\left(x_{1, j}\right)\left[1-F\left(x_{1, j}\right)\right]^{m-1}, \\
& F_{1: m}\left(x_{1, j}\right)=1-\left[1-F\left(x_{1, j}\right)\right]^{m}, \\
& f_{m: m}\left(x_{k}\right)=m f\left(x_{m, k}\right)\left[F\left(x_{m, k}\right)\right]^{m-1} \text { and } \quad F_{m: m}\left(x_{m, k}\right)= \\
& {\left[F\left(x_{m, k}\right)\right]^{m}}
\end{aligned}
$$

\section{Case II: $m$ odd $(m=2 r+1)$}

$$
\begin{aligned}
L(\theta) \\
=\left[\prod_{j=1}^{r} m f_{1: m}\left(x_{1, j}\right)\left[1-F_{1: m}\left(x_{1, j}\right)\right]^{m-1}\right] \\
\cdot\left[\prod_{k=r+2}^{m} m f_{m: m}\left(x_{m, k}\right)\left[F_{m: m}\left(x_{m, k}\right)\right]^{m-1}\right] \\
\cdot\left[\frac{(2 r+1) !}{(r) !(r) !} f_{r+1: m}\left(x_{(r+1),(r+1)}\right)\right. \\
\left.\left(F_{r+1: m}\left(x_{(r+1),(r+1)}\right)\left(1-F_{r+1: m}\left(x_{(r+1),(r+1)}\right)\right)\right)^{r}\right]
\end{aligned}
$$

where

$$
\begin{aligned}
F_{r+1: m}\left(x_{(r+1),(r+1)}\right) & \\
= & \sum_{t=r+1}^{m}\left(\begin{array}{c}
m \\
t
\end{array}\right)\left(F\left(x_{(r+1),(r+1)}\right)\right)^{t} \\
& \cdot\left(1-F\left(x_{(r+1),(r+1)}\right)\right)^{m-t}
\end{aligned}
$$

\section{Estimation of Three-Parameters Weibull Distribution Parameters}

This MLE method has a convergence issue and it can also have an unfeasible value so that the location estimate of the three-parameter Weibull model can be greater than the minimum value of the observations . also pointed out that the likelihood function has the unbounded likelihood problem and the location parameter tends to approach the smallest observation. also showed that no stationary point can yield a consistent estimator, which results in no local maximum. Thus, whether a global or a local maximum is sought, the MLE is bound to fail. So we are going to differentiate $\lambda$ and $\beta$ only.

In this Section MLEs for the unknown parameters of EPGW distribution based on RSS will be reviewed, moreover we will derive MLHs for EPGW distribution based on ERSS and DRSS.

\subsection{Estimation Based on RSS}

Let $\left\{X_{i}^{j}, i=1,2, \ldots, n ; j=1,2, \ldots, r\right\}$ be a ranked set sample with CDF and PDF given in Eq. (1) and Eq. (2), where $n$ is the set size, $r$ is the number of cycles and $m=$ $n r$. According to the Eq. (4) the Likelihood function for set sizes $m$ and with $r$ cycles based on RSS is given by

$$
\begin{aligned}
L_{r}(x ; \lambda, \beta, \alpha)=\prod_{j=1}^{r} & \prod_{i=1}^{n} C_{i} \lambda \beta\left(x_{(i) j}-\alpha\right)^{\beta-1} e^{-\lambda\left(x_{(i) j}-\alpha\right)^{\beta}} \\
& \cdot\left(1-e^{-\lambda\left(x_{(i) j}-\alpha\right)^{\beta}}\right)^{i-1}\left(e^{-\lambda\left(x_{(i) j}-\alpha\right)^{\beta}}\right)^{n-i}
\end{aligned}
$$

The log likelihood function can be derived directly as follows

$$
\begin{aligned}
\ell_{r}(\theta) \propto n r \log \lambda+ & n r \log \beta+(\beta-1) \sum_{j=1}^{r} \sum_{i=1}^{n} \log \left(x_{(i) j}-\alpha\right) \\
& +\sum_{j=1}^{r} \sum_{i=1}^{n}\left[-\lambda\left(x_{(i) j}-\alpha\right)^{\beta}\right] \\
& +\sum_{j=1}^{r} \sum_{i=1}^{n}(i-1) \log \left[1-e^{-\lambda\left(x_{(i) j}-\alpha\right)^{\beta}}\right] \\
& +\sum_{j=1}^{r} \sum_{i=1}^{n}(n-i)\left[-\lambda\left(x_{(i) j}-\alpha\right)^{\beta}\right]
\end{aligned}
$$

The likelihood equations becomes

$$
\begin{aligned}
\frac{\partial \ell_{E(e)}}{\partial \lambda}=\frac{n r}{\lambda}-\sum_{j=1}^{r} & \sum_{i=1}^{n}\left[\left(x_{(i) j}-\alpha\right)^{\beta}\right] \\
& +\sum_{j=1}^{r} \sum_{i=1}^{n}(i-1) \frac{e^{-\lambda\left(x_{(i) j}-\alpha\right)^{\beta}}\left(x_{(i) j}-\alpha\right)^{\beta}}{1-e^{-\lambda\left(x_{(i) j}-\alpha\right)^{\beta}}} \\
& -\sum_{j=1}^{r} \sum_{i=1}^{n}(n-i)\left[\left(x_{(i) j}-\alpha\right)^{\beta}\right]
\end{aligned}
$$

and

$$
\begin{aligned}
& \frac{\partial \ell_{E(e)}}{\partial \beta} \\
& =\frac{n r}{\beta}+\sum_{j=1}^{r} \sum_{i=1}^{n} \log x_{(i) j} \\
& +\sum_{j=1}^{r} \sum_{i=1}^{n}\left[-\lambda\left(x_{(i) j}-\alpha\right)^{\beta}\right] \log \left(x_{(i) j}-\alpha\right) \\
& +\sum_{j=1}^{r} \sum_{i=1}^{n}(i-1) \frac{e^{-\lambda\left(x_{(i) j}-\alpha\right)^{\beta}} \lambda\left(x_{(i) j}-\alpha\right)^{\beta} \log \left(x_{(i) j}-\alpha\right)}{1-e^{-\lambda\left(x_{(i) j}-\alpha\right)^{\beta}}} \\
& +\sum_{j=1}^{r} \sum_{i=1}^{n}(n-i)\left[-\lambda\left(x_{(i) j}-\alpha\right)^{\beta}\right] \log \left(x_{(i) j}-\alpha\right)
\end{aligned}
$$

\subsection{Estimation Based on ERSS}

The maximum likelihood function for even set sizes $(m=$ $2 p$ ) and with $r$ cycles by substitution in Eq. (9) based on ERSS is given by

$$
\begin{gathered}
L_{E(e)}(\theta) \propto \prod_{j=1}^{r} \prod_{i=1}^{p} m \lambda \beta\left(x_{(1) i, j}-\alpha\right)^{\beta-1}\left(\exp \left[-\lambda\left(x_{(1) i, j}-\alpha\right)^{\beta}\right]\right) \\
\cdot\left[1-e^{-\lambda\left(x_{(1) i j}-\alpha\right)^{\beta}}\right]^{m-1} \\
\cdot\left[\prod_{j=1}^{r} \prod_{i=p+1}^{m} m \lambda \beta\left(x_{(m) i, j}-\alpha\right)^{\beta-1}\left(\exp \left[-\lambda\left(x_{(m) i, j}-\alpha\right)^{\beta}\right]\right)^{m}\right]
\end{gathered}
$$




$$
\begin{aligned}
L_{E(e)}(\theta)=h m^{r m} \lambda^{r m} & \alpha^{r m} \beta^{r m}\left(\prod_{j=1}^{r} \prod_{i=1}^{p}\left(x_{(1) i, j}-\alpha\right)^{\beta-1}\right) \\
& \cdot\left(\prod_{j=1}^{r} \prod_{i=1}^{p} \exp \left[-\lambda\left(x_{(1) i, j}-\alpha\right)^{\beta}\right]\right) \\
& \cdot\left(\prod_{j=1}^{r} \prod_{i=1}^{p}\left[1-e^{-\lambda\left(x_{(1) i, j}-\alpha\right)^{\beta}}\right]^{m-1}\right) \\
& \cdot\left(\prod_{i=1}^{r} \prod_{j=p+1}^{m}\left(x_{(m) i, j}-\alpha\right)^{\beta-1}\right) \\
& \cdot\left(\prod_{j=1}^{r} \prod_{i=p+1}^{m}\left(\exp \left[-\lambda\left(x_{(m) i, j}-\alpha\right)^{\beta}\right]\right)^{m}\right)
\end{aligned}
$$

$\ell_{E(e)}(\theta)=\log h+r m \log m+r m \log \lambda+r m \log \beta$

$$
\begin{aligned}
& +(\beta-1) \sum_{j=1}^{r} \sum_{i=1}^{p} \log \left(x_{(1) i, j}-\alpha\right) \\
& -\lambda \sum_{j=1}^{r} \sum_{i=1}^{p}\left[\left(x_{(1) i, j}-\alpha\right)^{\beta}\right] \\
& +\sum_{j=1}^{r} \sum_{i=1}^{p}(m-1) \log \left[1-e^{-\lambda\left(x_{(1) i, j}-\alpha\right)^{\beta}}\right] \\
& +(\beta-1) \sum_{j=1}^{r} \sum_{i=p+1}^{m} \log \left(x_{(m) i, j}-\alpha\right) \\
& -\lambda \sum_{j=1}^{r} \sum_{i=p+1}^{m} m\left(\left(x_{(m) i, j}-\alpha\right)^{\beta}\right)
\end{aligned}
$$

and the likelihood equations are given by

$$
\begin{aligned}
\frac{\partial \ell_{E(e)}}{\partial \lambda}=\frac{r m}{\lambda}-\sum_{j=1}^{r} & \sum_{i=1}^{p}\left[\left(x_{(1) i, j}-\alpha\right)^{\beta}\right] \\
& +\sum_{j=1}^{r} \sum_{i=1}^{p}(m-1) \frac{e^{-\lambda\left(x_{(1) i, j}-\alpha\right)^{\beta}}\left(x_{(1) i, j}-\alpha\right)^{\beta}}{\left(1-e^{-\lambda\left(x_{(1) i, j}-\alpha\right)^{\beta}}\right)} \\
& -\sum_{j=1}^{r} \sum_{i=p+1}^{m} m\left(\left(x_{(m) i, j}-\alpha\right)^{\beta}\right)
\end{aligned}
$$

and

$$
\begin{aligned}
& \frac{\partial \ell_{E(e)}}{\partial \beta} \\
& =\frac{r m}{\beta}+\sum_{j=1}^{r} \sum_{i=1}^{p} \log \left(x_{(1) i, j}-\alpha\right) \\
& -\lambda \sum_{j=1}^{r} \sum_{i=1}^{p}\left[\left(x_{(1) i, j}-\alpha\right)^{\beta}\right] \log \left(x_{(1) i, j}-\alpha\right) \\
& +\sum_{j=1}^{r} \sum_{i=1}^{p}(m-1) \frac{e^{-\lambda\left(x_{(1) i, j}-\alpha\right)^{\beta}} \lambda\left(x_{(1) i, j}-\alpha\right)^{\beta} \log \left(x_{(1) i, j}-\alpha\right)}{\left(1-e^{-\lambda\left(x_{(1) i, j}-\alpha\right)^{\beta}}\right)} \\
& +\sum_{j=1}^{r} \sum_{i=p+1}^{m} \log \left(x_{(m) i, j}-\alpha\right) \\
& -\lambda \sum_{j=1}^{r} \sum_{i=p+1}^{m} m\left(\left(x_{(m) i, j}-\alpha\right)^{\beta} \log \left(x_{(m) i, j}-\alpha\right)\right)
\end{aligned}
$$

When $m$ is odd $(m=2 q+1)$ and based on ERSS by substitution in Eq. (8), the maximum likelihood function $L$ is given by

$$
\begin{aligned}
& L_{E(o)}(\theta) \propto \prod_{j=1}^{r} \prod_{i=1}^{q} m \lambda \beta\left(x_{(1) i, j}-\alpha\right)^{\beta-1} \cdot\left(\exp \left[-\lambda\left(x_{(1) i, j}-\alpha\right)^{\beta}\right]\right) \\
& \cdot\left[1-e^{-\lambda\left(x_{(1) i, j}-\alpha\right)^{\beta}}\right]^{m-1} \\
& \cdot\left[\prod_{j=1}^{r} \prod_{i=q+1}^{m-1} m \lambda \beta\left(x_{(m) i, j}-\alpha\right)^{\beta-1}\left(\exp \left[-\lambda\left(x_{(m) i, j}-\alpha\right)^{\beta}\right]\right)^{m}\right] \\
& \cdot\left[\left(\lambda \beta\left(x_{((m+1) / 2) j}-\alpha\right)^{\beta-1}\left(\exp \left[-\lambda\left(x_{((m+1) / 2) j}-\alpha\right)^{\beta}\right]\right)\right)\right. \\
& \left.\cdot\left[1-e^{-\lambda\left(x_{((m+1) / 2) j-\alpha)^{\beta}}\right.}\right]^{\frac{m-1}{2}}\left(\exp \left[-\lambda\left(x_{((m+1) / 2) j}-\alpha\right)^{\beta}\right]\right)^{\frac{m-1}{2}}\right] \\
& \ell_{E(e)}(\theta)=\log k+r m \log m+r m \log \lambda+r m \log \beta \\
& +(\beta-1) \sum_{j=1}^{r} \sum_{i=1}^{q} \log \left(x_{(1) i, j}-\alpha\right)-\lambda \sum_{j=1}^{r} \sum_{i=1}^{q}\left[\left(x_{(1) i, j}-\alpha\right)^{\beta}\right] \\
& +\sum_{j=1}^{r} \sum_{i=1}^{q}(m-1) \log \left[1-e^{-\lambda\left(x_{(1) i, j}-\alpha\right)^{\beta}}\right] \\
& +(\beta-1) \sum_{j=1}^{r} \sum_{i=q+1}^{m-1} \log \left(x_{(m) i, j}-\alpha\right) \\
& -\lambda \sum_{j=1}^{r} \sum_{i=q+1}^{m-1} m\left(\left(x_{(m) i, j}-\alpha\right)^{\beta}\right)+(\beta-1) \log \left(x_{\left(\frac{m+1}{2}\right) j}-\alpha\right) \\
& +\frac{m-1}{2} \log \left(1-e^{-\lambda\left(x_{\left.((m+1) / 2) j^{-\alpha}\right)^{\beta}}\right.}\right) \\
& +\left(\frac{m+1}{2}\right)\left[-\lambda\left(x_{((m+1) / 2) j}-\alpha\right)^{\beta}\right]
\end{aligned}
$$

and the likelihood equations are given by

$$
\begin{aligned}
& \frac{\partial \ell_{E(e)}}{\partial \lambda}=\frac{r m}{\lambda}-\sum_{j=1}^{r} \sum_{i=1}^{q}\left[\left(x_{(1) i, j}-\alpha\right)^{\beta}\right] \\
& +\sum_{j=1}^{r} \sum_{i=1}^{q}(m-1) \frac{e^{-\lambda\left(x_{(1) i, j}-\alpha\right)^{\beta}}\left(x_{(1) i, j}-\alpha\right)^{\beta}}{\left(1-e^{-\lambda\left(x_{(1) i, j}-\alpha\right)^{\beta}}\right)} \\
& -\sum_{j=1}^{r} \sum_{i=q+1}^{m-1} m\left(\left(x_{(m) i, j}-\alpha\right)^{\beta}\right) \\
& +\left(\frac{m-1}{2}\right) \frac{e^{-\lambda\left(x_{\left(\frac{m+1}{2}\right) j}-\alpha\right)^{\beta}}\left(x_{\left(\frac{m+1}{2}\right) j}-\alpha\right)^{\beta}}{1-e^{-\lambda\left(x\left(\frac{m+1}{2}\right) j^{-\alpha)^{\beta}}\right.}} \\
& -\left(\frac{m+1}{2}\right)\left[\left(x_{((m+1) / 2) j}-\alpha\right)^{\beta}\right]
\end{aligned}
$$

and

$\frac{\partial \ell_{E(e)}}{\partial \beta}$

$=\frac{r m}{\beta}+\sum_{j=1}^{r} \sum_{i=1}^{q} \log \left(x_{(1) i, j}-\alpha\right)-\lambda \sum_{j=1}^{r} \sum_{i=1}^{q}\left[\left(x_{(1) i, j}-\alpha\right)^{\beta}\right] \log \left(x_{(1) i, j}-\alpha\right)$

$+\sum_{j=1}^{r} \sum_{i=1}^{q}(m-1) \frac{e^{-\lambda\left(x_{(1) i, j}-\alpha\right)^{\beta}} \lambda\left(x_{(1) i, j}-\alpha\right)^{\beta} \log \left(x_{(1) i, j}-\alpha\right)}{\left(1-e^{-\lambda\left(x_{(1) i, j}-\alpha\right)^{\beta}}\right)}$

$+\sum_{j=1}^{r} \sum_{i=q+1}^{m-1} \log \left(x_{(m) i, j}-\alpha\right)-\lambda \sum_{j=1}^{r} \sum_{i=q+1}^{m-1} m\left(\left(x_{(m) i, j}-\alpha\right)^{\beta} \log \left(x_{(m) i, j}-\alpha\right)\right)$

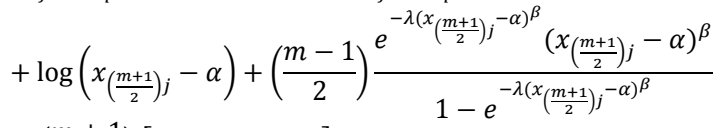

$-\left(\frac{m+1}{2}\right)\left[\left(x_{\left(\frac{m+1}{2}\right) j}-\alpha\right)^{\beta}\right]$

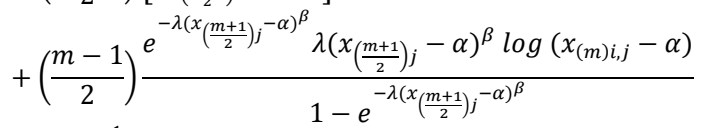

$+\left(\frac{m+1}{2}\right)\left[-\lambda\left(x_{\left(\frac{m+1}{2}\right) j}-\alpha\right)^{\beta}\right] \log \left(x_{(m) i, j}-\alpha\right)$ 


\subsection{Estimation Based on DRSS}

By substitution in Eq. (10) and Eq. (11) based on EPGW distribution the Likelihood function for set sizes $m$ and with $r$ cycles based on DRSS is given by

\section{Case I: $m$ even $(m=2 r)$}

$$
\begin{array}{r}
L_{D(e)}(\theta)=\left[\prod_{j=1}^{r}\left(m^{2} \lambda \beta\left(x_{(1) i, j}-\alpha\right)^{\beta-1}\left(e^{-\lambda\left(x_{(1) i, j}-\alpha\right)^{\beta}}\right)^{m^{2}}\right)\right] \\
\cdot \prod_{k=r+1}^{m}\left(m^{2} \lambda \beta\left(x_{(m) i, j}-\alpha\right)^{\beta-1} \exp \left[-\lambda\left(x_{(m) i, j}-\alpha\right)^{\beta}\right]\right) \\
\cdot\left(1-e^{-\lambda\left(x_{(m) i, j}-\alpha\right)^{\beta}}\right)^{m^{2}-1}
\end{array}
$$

Then, the associated log-likelihood function is obtained as

$$
\begin{aligned}
\ell_{D(e)}=2 m \log m+ & m \log \lambda+m \log \beta \\
& +(\beta-1) \sum_{j=1}^{r} \log \left(x_{(1) i, j}-\alpha\right) \\
& +(\beta-1) \sum_{k=r+1}^{m} \log \left(x_{(m) i, j}-\alpha\right) \\
& +m^{2} \sum_{j=1}^{r}\left(-\lambda\left(x_{(1) i, j}-\alpha\right)^{\beta}\right) \\
& +\sum_{k=r+1}^{m}\left(-\lambda\left(x_{(m) i, j}-\alpha\right)^{\beta}\right) \\
& +\left(m^{2}-1\right) \sum_{k=r+1}^{m} \log \left(1-e^{-\lambda\left(x_{(m) i, j}-\alpha\right)^{\beta}}\right) .
\end{aligned}
$$

and the likelihood equations are given by

$$
\begin{aligned}
\frac{\partial \ell_{D(e)}}{\partial \lambda}=\frac{m}{\lambda}-m^{2} \sum_{j=1}^{r}\left(\left(x_{(1) i, j}-\alpha\right)^{\beta}\right) & \\
& -\sum_{k=r+1}^{m}\left(\left(x_{(m) i, j}-\alpha\right)^{\beta}\right) \\
& +\left(m^{2}-1\right) \sum_{k=r+1}^{m} \frac{e^{-\lambda\left(x_{(m) i, j}-\alpha\right)^{\beta}}\left(x_{(m) i, j}-\alpha\right)^{\beta}}{\left(1-e^{-\lambda\left(x_{(m) i, j}-\alpha\right)^{\beta}}\right)}
\end{aligned}
$$

and

$$
\begin{aligned}
\frac{\partial \ell_{D(e)}}{\partial \beta} & =\frac{m}{\beta}+\sum_{j=1}^{r} \log \left(x_{(1) i, j}-\alpha\right)+\sum_{k=r+1}^{m} \log \left(x_{(m) i, j}-\alpha\right) \\
& +m^{2} \sum_{j=1}^{r}\left(-\lambda\left(x_{(1) i, j}-\alpha\right)^{\beta}\right) \log \left(x_{(1) i, j}-\alpha\right) \\
& +\sum_{k=r+1}^{m}\left(-\lambda\left(x_{(m) i, j}-\alpha\right)^{\beta}\right) \log \left(x_{(m) i, j}-\alpha\right) \\
+\left(m^{2}-1\right) & \sum_{k=r+1}^{m} \frac{e^{-\lambda\left(x_{(m) i, j}-\alpha\right)^{\beta}} \lambda\left(x_{(m) i, j}-\alpha\right)^{\beta} \log \left(x_{(m) i, j}-\alpha\right)}{\left(1-e^{-\lambda\left(x_{(m) i, j}-\alpha\right)^{\beta}}\right)}
\end{aligned}
$$

\section{Case II: $m$ odd $(m=2 r+1)$}

$$
\begin{gathered}
L_{D(o)}(\theta)=\left[\prod_{j=1}^{r}\left(m^{2} \lambda \beta\left(x_{(1) i, j}-\alpha\right)^{\beta-1}\left(e^{-\lambda\left(x_{(1) i, j}-\alpha\right)^{\beta}}\right)^{m^{2}}\right)\right] \\
\cdot \prod_{k=r+2}^{m}\left(m^{2} \lambda \beta\left(x_{(m) i, j}-\alpha\right)^{\beta-1} \exp \left[-\lambda\left(x_{(m) i, j}-\alpha\right)^{\beta}\right]\right) \\
\cdot\left(1-e^{-\lambda\left(x_{(m) i j}-\alpha\right)^{\beta}}\right)^{m^{2}-1} \\
\cdot\left[\frac{(2 r+1) !}{(r) !(r) !}\left(\frac{m !}{r ! r !}\left(\lambda \beta\left(x_{(r+1),(r+1)}-\alpha\right)^{\beta-1}\left(\exp \left[-\lambda\left(x_{(r+1),(r+1)}-\alpha\right)^{\beta}\right]\right)^{(r+1)}\right)\right)\right. \\
\cdot\left(\left(1-e^{-\lambda\left(x_{(r+1),(r+1)}-\alpha\right)^{\beta}}\right)^{r}\right) \\
\cdot\left(F_{r+1: m}\left(x_{(r+1),(r+1)}\right)\right)^{r}\left(1-F_{r+1: m}\left(x_{(r+1),(r+1)}\right)\right)^{r}
\end{gathered}
$$

Then, the associated log-likelihood function is obtained as

$$
\begin{aligned}
\ell_{D(e)}=(2 m-1) & \log m+m \log \lambda+m \log \beta \\
& +(\beta-1) \sum_{j=1}^{r} \log \left(x_{(1) i, j}-\alpha\right) \\
& +(\beta-1) \sum_{k=r+2}^{m} \log \left(x_{(m) i, j}-\alpha\right) \\
& +m^{2} \sum_{j=1}^{r}\left(-\lambda\left(x_{(1) i, j}-\alpha\right)^{\beta}\right) \\
& +\sum_{k=r+2}^{m}\left(-\lambda\left(x_{(m) i, j}-\alpha\right)^{\beta}\right) \\
& +\left(m^{2}-1\right) \sum_{k=r+2}^{m} \log \left(1-e^{-\lambda\left(x_{(m) i, j}-\alpha\right)^{\beta}}\right) \\
& +(\beta-1) \log \left(x_{(r+1),(r+1)}-\alpha\right) \\
& +(r+1)\left[-\lambda\left(x_{(r+1),(r+1)}-\alpha\right)^{\beta}\right] \\
& +r \log \left(1-e^{-\lambda\left(x_{(r+1),(r+1)}-\alpha\right)^{\beta}}\right) \\
& +r \log F_{r+1: m}\left(x_{(r+1),(r+1)}\right) \\
& +r \log \left(1-F_{r+1: m}\left(x_{(r+1),(r+1)}\right)\right)
\end{aligned}
$$

and the likelihood equations are given by

$\frac{\partial \ell_{D(e)}}{\partial \lambda}$

$=\frac{m}{\lambda}-m^{2} \sum_{j=1}^{r}\left(\left(x_{(1) i, j}-\alpha\right)^{\beta}\right)-\sum_{k=r+1}^{m}\left(\left(x_{(m) i, j}-\alpha\right)^{\beta}\right)+\left(m^{2}\right.$

- 1) $\sum_{k=r+2}^{m} \frac{e^{-\lambda\left(x_{(m) i, j}-\alpha\right)^{\beta}}\left(x_{(m) i, j}-\alpha\right)^{\beta}}{\left(1-e^{-\lambda\left(x_{(m) i, j}-\alpha\right)^{\beta}}\right)}-(r$

$+1)\left[\left(x_{(r+1),(r+1)}-\alpha\right)^{\beta}\right]$

$+r\left(\frac{e^{-\lambda\left(x_{(r+1),(r+1)}-\alpha\right)^{\beta}}\left(x_{(r+1),(r+1)}-\alpha\right)^{\beta}}{1-e^{-\lambda\left(x_{(r+1),(r+1)}-\alpha\right)^{\beta}}}\right)$

$+F_{\lambda}\left(\frac{1-2 F_{r+1: m}\left(x_{(r+1),(r+1)}\right)}{F_{r+1: m}\left(x_{(r+1),(r+1)}\right)\left(1-F_{r+1: m}\left(x_{(r+1),(r+1)}\right)\right)}\right)$

and 
$\frac{\partial \ell_{D(e)}}{\partial \beta}$

$=\frac{m}{\beta}+\sum_{j=1}^{r} \log \left(x_{(1) i, j}-\alpha\right)+\sum_{k=r+1}^{m} \log \left(x_{(m) i, j}-\alpha\right)$

$+m^{2} \sum_{j=1}^{r}\left(-\lambda\left(x_{(1) i, j}-\alpha\right)^{\beta}\right) \log \left(x_{(1) i, j}-\alpha\right)$

$+\sum_{k=r+2}^{m}\left(-\lambda\left(x_{(m) i, j}-\alpha\right)^{\beta}\right) \log \left(x_{(m) i, j}-\alpha\right)$

$+\left(m^{2}-1\right) \sum_{k=r+2}^{m} \frac{e^{-\lambda\left(x_{(m) i, j}-\alpha\right)^{\beta}} \lambda\left(x_{(m) i, j}-\alpha\right)^{\beta} \log \left(x_{(m) i, j}-\alpha\right)}{\left(1-e^{-\lambda\left(x_{(m) i, j}-\alpha\right)^{\beta}}\right)}$

$+\log \left(x_{(r+1),(r+1)}-\alpha\right) \quad+(r$

$+1)\left[-\lambda\left(x_{(r+1),(r+1)}-\alpha\right)^{\beta}\right] \log \left(x_{(r+1),(r+1)}-\alpha\right)$

$+r\left(\frac{e^{-\lambda\left(x_{(r+1),(r+1)}-\alpha\right)^{\beta}} \lambda\left(x_{(r+1),(r+1)}-\alpha\right)^{\beta} \log \left(x_{(r+1),(r+1)}-\alpha\right)}{1-e^{-\lambda\left(x_{(r+1),(r+1)}-\alpha\right)^{\beta}}}\right)$

$+F_{\beta}\left(\frac{1-2 F_{r+1: m}\left(x_{(r+1),(r+1)}\right)}{F_{r+1: m}\left(x_{(r+1),(r+1)}\right)\left(1-F_{r+1: m}\left(x_{(r+1),(r+1)}\right)\right)}\right)$

\section{Simulation Study}

In this section, a simulation study will be conducted to compare the maximum likelihood estimates of the ThreeParameters Weibull distribution ratio and shape parameters based on different sampling schemes. The simulation is suitable for 10,000 repetitions and different sample sizes, $\mathrm{m}$ $=\{10,15,20,25\}$. For different parameter values EPGW $(\lambda$, $\beta)=\{(0.5,0.5),(0.5,1.5),(1.5,1.5)\}$ for simulation of the statistical software R [20]. Use MSE and efficiency standards to compare the $\lambda$ and $\beta$ estimators proposed using SRS, RSS, ERSS and DRSS. Calculate the efficiency of all estimators based on SRS relative to MLE. The efficiency of the estimator is defined as

$$
\operatorname{eff}\left(\hat{\theta}_{1}, \hat{\theta}_{2}\right)=\frac{\operatorname{MSE}\left(\hat{\theta}_{1}\right)}{\operatorname{MSE}\left(\hat{\theta}_{2}\right)}
$$

if $\operatorname{eff}\left(\hat{\theta}_{1}, \hat{\theta}_{2}\right)>1$, then $\hat{\theta}_{2}$ is better than $\hat{\theta}_{1}$.

Table 1and Table 2 lists the results of bias and MSE for different estimators, table (3) lists the efficiency results, and Figure (6-11) shows the simulation results. The following conclusions can be observed from Table (1-2)

1) It is small biases in almost all cases

2) The MSE of the $(\lambda, \beta)$ estimator based on SRS data is greater than the MSE of the estimator based on RSS, ERSS and DRSS data in all cases. (See Figure 6).

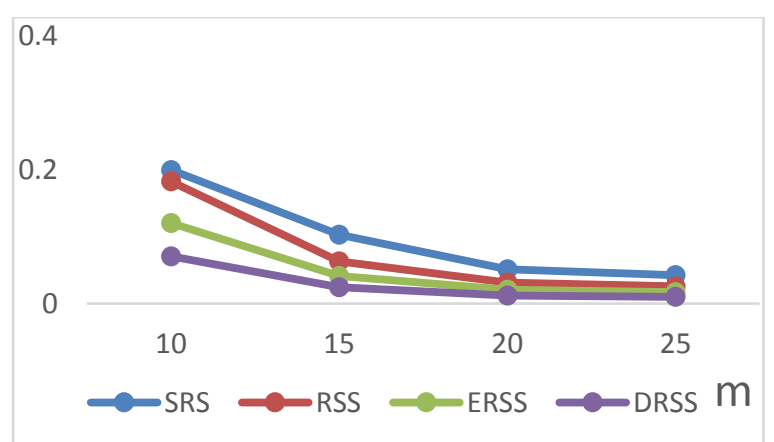

Figure 6. MSEs of the estimators for $\lambda=0.5$
3) As the set size increases, the MSE of all estimators based on SRS, RSS, ERSS and DRSS will decrease in almost all cases. (See Figure 7).

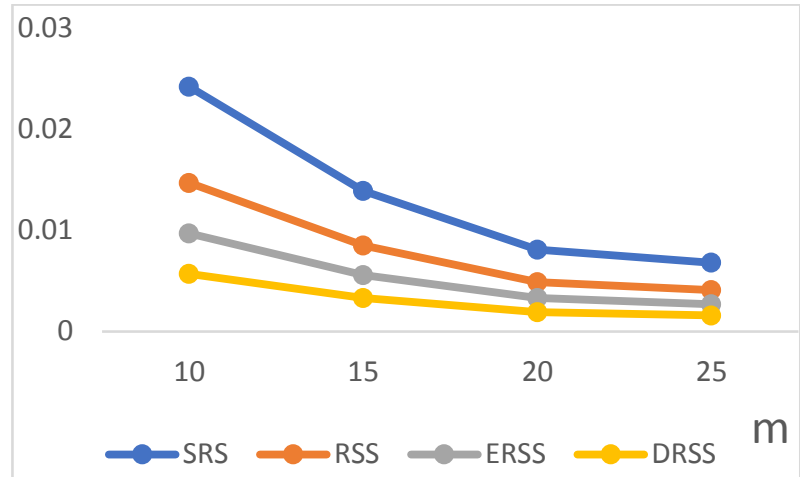

Figure 7. MSEs of the estimators based on SRS, RSS, ERSS, and DRSS at $(\beta=0.5)$

4) the MSE of all estimators based on SRS, RSS, ERSS and DRSS decreases as the value of $\lambda$ increases (see Figure 8 ). As the value of $\beta$ increases, the MSE of all estimates based on SRS, RSS, ERSS and DRSS will decrease (see Figure 9).MSEs of the estimators for $(\lambda)$ based on DRSS have the smallest

5) In almost all cases, The MSEs of the MRSS scheme based on estimators ( $\lambda$ and $\beta$ ) are smaller than the MSEs of the RSS estimators.

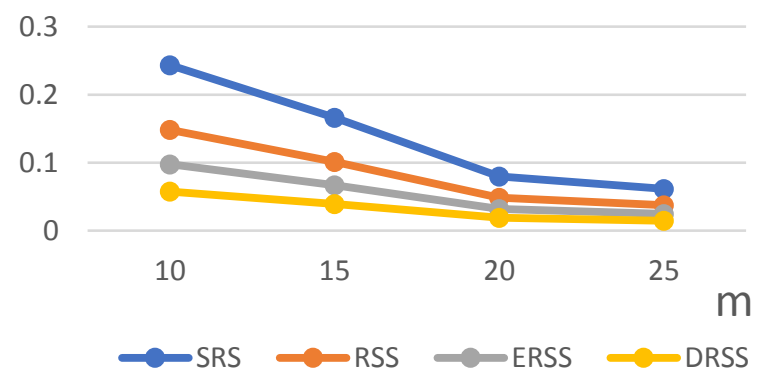

Figure 8. MSEs of the estimators based on SRS, RSS, ERSS, and DRSS at $\lambda=1.5$

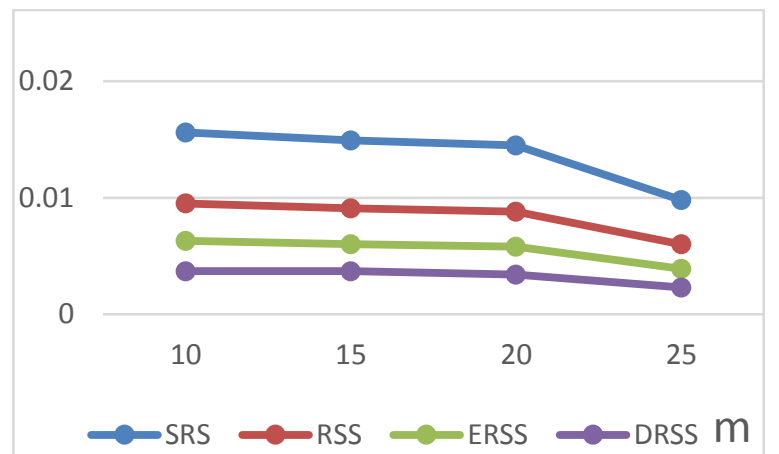

Figure 9. MSEs of the estimators based on SRS, RSS, ERSS, and DRSS at $\beta=1.5$

From Table 3, it can be observed that: 
6) As the set size increases, the efficiency of all estimators based on RSS, ERSS and DRSS will increase in almost all cases. (See Figure 10).

7) in all cases, Efficiencies of the estimators for $\lambda$ based on DRSS has the highest efficiency, (See Figure 10).

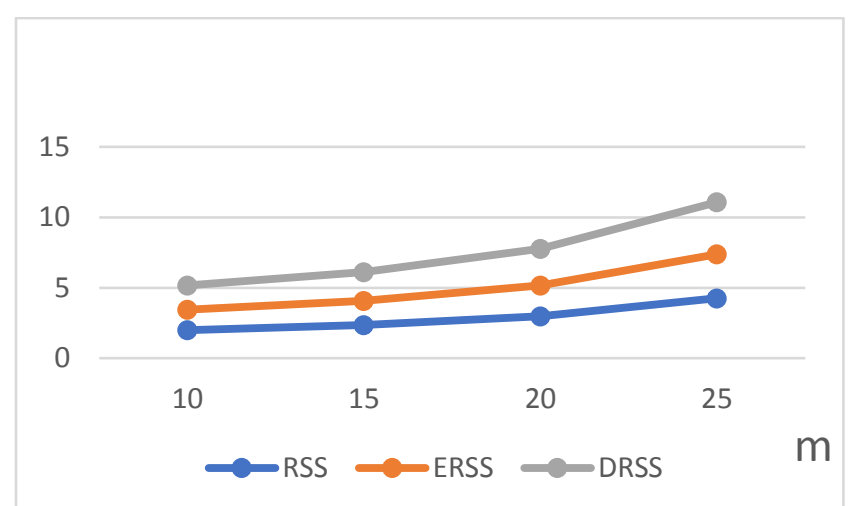

Figure 10. Efficiencies of the estimators based on RSS, ERSS, and DRSS at $\lambda=0.5$
8) in all cases, Efficiencies of the estimators for $\beta$ based on DRSS have the largest efficiencies (See Figure 11).

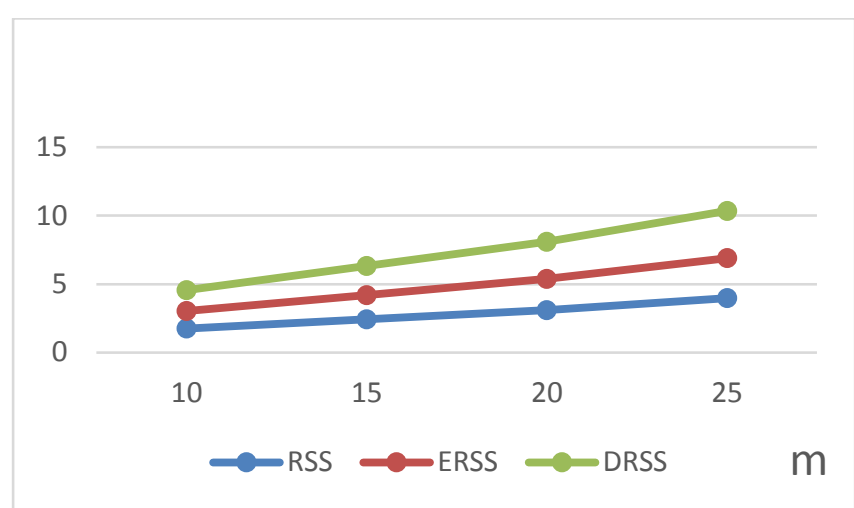

Figure 11. Efficiencies of the estimators based on RSS, ERSS, and DRSS at $\beta=0.5$

9) In almost all cases, The efficiency of ( $\lambda$ and $\propto$ ) estimators on ERSS is greater than that of RSS estimators.

Table 1. Biases of the estimators for $(\lambda, \beta)$ based on SRS, RSS, ERSS, and DRSS

\begin{tabular}{|c|c|c|c|c|c|c|c|c|c|}
\hline \multirow{2}{*}{$\begin{array}{l}\text { Three- } \\
\text { Parameters } \\
\text { Weibull }(\lambda, \beta)\end{array}$} & \multirow{2}{*}{$\mathrm{m}$} & \multicolumn{2}{|l|}{ SRS } & \multicolumn{2}{|l|}{ RSS } & \multicolumn{2}{|l|}{ MRSS } & \multicolumn{2}{|l|}{ NRSS } \\
\hline & & $\lambda$ & $\beta$ & $\lambda$ & $\beta$ & $\lambda$ & $\beta$ & $\lambda$ & $\beta$ \\
\hline \multirow{4}{*}{$(0.5,0.5)$} & 10 & -0.3290 & -0.9610 & -0.0923 & 0.4495 & 0.3330 & 0.0840 & -0.2387 & -0.1775 \\
\hline & 15 & -0.1080 & -0.7755 & -0.0791 & 0.4014 & 0.2357 & -0.0696 & -0.2189 & -0.1639 \\
\hline & 20 & -0.0887 & -0.6325 & -0.0477 & 0.2472 & 0.1591 & -0.0430 & -0.1837 & -0.1031 \\
\hline & 25 & -0.0440 & -0.1280 & -0.4442 & 0.1939 & 0.1251 & -0.0273 & -0.1530 & -0.0808 \\
\hline \multirow{4}{*}{$(0.5,1.5)$} & 10 & -0.3948 & -1.1532 & -0.1107 & 0.5394 & 0.3996 & 0.1008 & -0.2864 & -0.213 \\
\hline & 15 & -0.1296 & -0.9306 & -0.0949 & 0.48168 & 0.28284 & -0.0832 & -0.2626 & -0.1968 \\
\hline & 20 & -0.1064 & -0.759 & -0.0572 & 0.29664 & 0.1902 & -0.0516 & -0.2204 & -0.1372 \\
\hline & 25 & -0.0528 & -0.1536 & -0.5334 & 0.23268 & 0.1512 & -0.0326 & -0.183 & -0.0996 \\
\hline \multirow{4}{*}{$(1.5,1.5)$} & 10 & -0.2278 & -0.6653 & -0.0639 & 0.3112 & 0.2305 & 0.0582 & -0.1653 & -0.1229 \\
\hline & 15 & -0.0748 & -0.5369 & -0.0548 & 0.2779 & 0.1632 & -0.0482 & -0.1515 & -0.1135 \\
\hline & 20 & -0.0614 & -0.4379 & -0.0330 & 0.1711 & 0.1101 & -0.0298 & -0.1272 & -0.0714 \\
\hline & 25 & -0.0305 & -0.0886 & -0.3075 & 0.1342 & 0.0866 & -0.0189 & -0.1059 & -0.0559 \\
\hline
\end{tabular}

Table 2. MSEs of the estimators for $(\lambda, \beta)$ based on SRS, RSS, ERSS, and DRSS

\begin{tabular}{|c|c|c|c|c|c|c|c|c|c|}
\hline \multirow{2}{*}{$\begin{array}{l}\text { Three- } \\
\text { Parameters } \\
\text { Weibull }\end{array}$} & \multirow{2}{*}{$\mathrm{m}$} & \multicolumn{2}{|l|}{ SRS } & \multicolumn{2}{|l|}{ RSS } & \multicolumn{2}{|l|}{ MRSS } & \multicolumn{2}{|l|}{ NRSS } \\
\hline & & $\lambda$ & $\beta$ & $\lambda$ & $\beta$ & $\lambda$ & $\beta$ & $\lambda$ & $\beta$ \\
\hline \multirow{4}{*}{$\begin{array}{l}\text { EPGW } \\
(0.5,0.5)\end{array}$} & 10 & 0.1999 & 0.0242 & $(0.5,0.5)$ & 0.0147 & 0.1204 & 0.0097 & 0.0707 & 0.0057 \\
\hline & 15 & 0.1033 & 0.0139 & 0.0629 & 0.0085 & 0.0415 & 0.0056 & 0.0243 & 0.0033 \\
\hline & 20 & 0.0512 & 0.0081 & 0.0312 & 0.0049 & 0.0205 & 0.0033 & 0.0121 & 0.0019 \\
\hline & 25 & 0.0424 & 0.0068 & 0.0258 & 0.0041 & 0.0170 & 0.0027 & 0.0100 & 0.0016 \\
\hline \multirow{4}{*}{$\begin{array}{l}\text { EPGW } \\
(0.5,1.5)\end{array}$} & 10 & 0.1683 & 0.0156 & $(0.5,1.5)$ & 0.0095 & 0.0675 & 0.0063 & 0.0397 & 0.0037 \\
\hline & 15 & 0.0924 & 0.0149 & 0.0563 & 0.0091 & 0.0371 & 0.0234 & 0.0218 & 0.0037 \\
\hline & 20 & 0.0283 & 0.0145 & 0.0172 & 0.0088 & 0.0114 & 0.0058 & 0.016 & 0.0034 \\
\hline & 25 & 0.0215 & 0.0098 & 0.0131 & 0.0060 & 0.0086 & 0.0039 & 0.0091 & 0.0023 \\
\hline \multirow{4}{*}{$\begin{array}{l}\text { EPGW } \\
(1.5,1.5)\end{array}$} & 10 & 0.2434 & 0.0395 & $(1.5,1.5)$ & 0.0240 & 0.0977 & 0.0159 & 0.0574 & 0.0093 \\
\hline & 15 & 0.1662 & 0.0293 & 0.1012 & 0.0178 & 0.0667 & 0.0118 & 0.0392 & 0.0069 \\
\hline & 20 & 0.0798 & 0.0259 & 0.0486 & 0.0158 & 0.0320 & 0.0104 & 0.0188 & 0.0061 \\
\hline & 25 & 0.06150 & 0.01870 & 0.03744 & 0.01139 & 0.02468 & 0.00751 & 0.0149 & 0.0044 \\
\hline
\end{tabular}


Table 3. Efficiencies of the estimators for $(\lambda, \beta)$ based on RSS, ERSS, and DRSS

\begin{tabular}{|c|c|c|c|c|c|c|c|}
\hline $\begin{array}{l}\text { Three- } \\
\text { Parameters } \\
\text { Weibull }(\lambda, \beta)\end{array}$ & $\mathrm{m}$ & RSS & $\beta$ & MRSS & $\beta$ & NRSS & $\beta$ \\
\hline \multirow{4}{*}{$\begin{array}{l}\text { EPGW } \\
(0.5,0.5)\end{array}$} & 10 & 1.6425 & $(0.5,0.5)$ & 2.8473 & 2.8473 & 4.2731 & 4.2758 \\
\hline & 15 & 2.3625 & 2.1013 & 4.0955 & 3.6427 & 6.1463 & 5.4703 \\
\hline & 20 & 3.2555 & 2.9855 & 5.6435 & 5.1754 & 8.4695 & 7.7721 \\
\hline & 25 & 3.8755 & 3.1435 & 6.7183 & 5.4493 & 10.0825 & 8.1834 \\
\hline \multirow{4}{*}{$\begin{array}{l}\text { EPGW } \\
(0.5,1.5)\end{array}$} & 10 & 1.9855 & $(0.5,1.5)$ & 3.4419 & 3.0371 & 5.1655 & 4.5609 \\
\hline & 15 & 2.3533 & 2.4253 & 4.0795 & 4.2043 & 6.1224 & 6.3137 \\
\hline & 20 & 2.9852 & 3.1112 & 5.1749 & 5.3933 & 7.7663 & 8.0993 \\
\hline & 25 & 4.2556 & 3.9755 & 7.3772 & 6.8916 & 11.0714 & 10.3493 \\
\hline \multirow{4}{*}{$\begin{array}{l}\text { EPGW } \\
(1.5,1.5)\end{array}$} & 10 & 2.2522 & $(1.5,1.5)$ & 3.9042 & 4.4347 & 5.8593 & 6.6597 \\
\hline & 15 & 2.8550 & 2.9862 & 4.9492 & 5.1767 & 7.4276 & 7.7739 \\
\hline & 20 & 3.2156 & 3.6578 & 5.5743 & 6.3409 & 8.3657 & 9.5222 \\
\hline & 25 & 3.9980 & 4.1690 & 6.9306 & 7.2271 & 10.4012 & 10.8530 \\
\hline
\end{tabular}

\section{Conclusions}

According to the numerical results, it can be concluded that compared with RSS and its modification schemes, MSEbased SRS data has the largest MSE. It can be noticed that in almost all cases, the MSE decreases as the setting size increases, and the efficiency increases as the setting size increases. This research shows that ERSS is better than RSS. Moreover, DRSS technology has advantages over other sampling schemes. In almost all cases, DRSS has the smallest MSE and the highest efficiency. Generally, estimators based on DRSS, EERS, and RSS based on RSS technology are more effective than estimators based on SRS technology.

\section{Conflict of Interest Statement}

The authors declare no conflict of interest.

\section{References}

[1] J. W. Pratt, Concavity of the log likelihood, J. Amer. Statist. Assoc 76 (1981).

[2] R. L. Smith, J. C. Naylor, A comparison of maximum likelihood and Bayesian estimators (1987)

[3] G. A. McIntyre, A method for unbiased selective sampling, using ranked sets, Aust. J. Agr. Res. 3 (1952) 385-390.

[4] H. M. Samawi, M. S. Ahmed, W. A. Abu-Dayyeh, (1996). Estimating the population mean using extreme ranked set sampling, The Biometrical Journal 38 (1996) 577-586.

[5] H. A. Muttlak, Median ranked set sampling, J. Appl. Stat. Sci. 6(1997) 245- 255.

[6] M. F. Al-Saleh, M. A. Al-Kadiri, Double ranked set sampling. Stat. Prob. Lett. 48 (2000) 205-212.

[7] K. Lam, B. K. Sinha, Z. Wu, Estimation of parameters in a two parameter exponential distribution using ranked set sampling, Ann. Inst. Statist. Math. 46 (1994) 723-736.

[8] A. I. Al-Omari, S. Al-Hadhrami, On Maximum Likelihood Estimators of the Parameters of a Modified Weibull Distribution Using Extreme Ranked Set Sampling, Journal of Modern Applied Statistical Methods 10 (2011) 607-617.
[9] M. A. Sabry, H.Z. Muhammed, A. Nabih, M. Shaaban, Parameter estimation for the power generalized Weibull distribution based on one- and two-stage ranked set sampling designs, J. Stat. Appl. Probab. 8 (2019) 113-128

[10] M. A. Sabry, M. Shaaban, Dependent Ranked Set Sampling Designs for Parametric Estimation with Applications, Ann. Data. Sci. 7 (2020) 357-371.

[11] M. Shaaban, Comparison Between Dependent and Independent Ranked Set Sampling Designs for Parametric Estimation with Applications, Ann. Data. Sci. (2020)

[12] C. A. Taconeli, A. S. Cabral, New two-stage sampling designs based on neoteric ranked set sampling, J. Stat. Comput. Simul. 89 (2018) 232-248

[13] F. G. Akgül, B. Seno $־$ glu, Estimation of $\mathrm{p}(\mathrm{x}<\mathrm{y})$ using ranked set sampling for theWeibull distribution, Qualit. Technol. Quantit. Manag. 14 (2017) 296-309

[14] L. Amro, M. H. Samuh, More powerful permutation test based on multistage ranked set sampling, Commun StatSimul Comput 46 (2017) 1-14

[15] C. N. Bouza-Herrera, A. I. F. Al-Omari, Ranked set sampling: 65 years improving the accuracy in data gathering, Academic Press, Cambridge (2018)

[16] M. Esemen, S. Gürler, Parameter estimation of generalized Rayleigh distribution based on ranked set sample, J Stat Comput Simul 88 (2018) 615-628

[17] L. Khan, J. Shabbir, Generalized exponential-type ratio-cum ratio estimators of population mean in ranked set and stratified ranked set sampling, Journal of Statistics and Management Systems 20 (2017) 133-151.

[18] J. Chakrabarty, S. Chowdhury, Compounded generalized Weibull distributions - A unified approach, Journal of Statistics and Management Systems 23 (2020) 887-913.

[19] L. Khan, J. Shabbir, A. Khalil, Modified ranked set sampling schemes for estimating the population mean, Journal of Statistics and Management Systems 22 (2019) 1481-1497.

[20] R. C. Team, R: A language and environment for statistical computing, R Foundation for Statistical Computing, Vienna, Austria (2017) https://www.R-project.org/ 